\title{
Treatment of corneal disease with traditional Tibetan medicine: A report of four cases
}

\author{
Tseten Yangkyi, $\mathrm{MD}^{1}$ and Stephen G Waller, $\mathrm{MD}^{2}$ \\ ${ }^{1}$ Traditional Tibetan Medicine Hospital (Menzikhang), Lhasa, TAR, PRC \\ ${ }^{2}$ Uniformed Services University of Health Sciences, Bethesda, MD, USA
}

\section{Introduction}

Traditional Tibetan medicine (TTM) has a long history on the Tibet plateau, stretching back at least 1500 years, drawn from its own traditions as well as Indian, Chinese, and recently, Western Medicine (WM). The physiologic and anatomic bases of WM are distinct from those used in TTM, consistent with its roots in the Buddhist faith. The treatment of common eye diseases, known as chi dip, has similar traditions. TTM has a large and loyal following among the Tibetan people, who are sparsely scattered on the high plains and mountain valleys of the Tibet Autonomous Region, People's Republic of China.

\section{Materials and methods}

With the hope of better mutual understanding of ophthalmologic practice, the second author has made several visits to the Menzikhang Hospital in Lhasa, where the first author is chief of the ophthalmology service. These visits have been sponsored by Seva Foundation, a humanitarian service organization in Berkeley, California, which has been supporting eye care in T.A.R. for more than a decade. Seva sponsored a Tibetan-Western ophthalmology conference in Dartsedo, Sichuan province, China, in 2000. The participants recommended WM-style study of TTM and the specific role of mig gi 'abedes sman bdud rtsi pha la (pha la for short), a powder applied directly to the eye for a broad spectrum of external diseases. This medication, which comes in

Received on: 04.07.2010 Accepted on: 17.11.2010

Address for correspondence

Uniformed Services University of Health Sciences,

Bethesda, MD, USA

Email: stevewaller@rcn.com;

cell 210-723-4339;

Fax 202-248-0502 a red and more potent white form, is used for six disorders in the Tibetan classification: shamdzir, corresponding to pterygium in WM; ling tok, leukoma (corneal scar, corneal ulcer, nodular keratopathy, band keratopathy, granular dystrophy, and mild versions of spheroidal degeneration); shalak, pinguecula and inflammatory disease of the nasal limbus; tsa drip, seasonal allergic conjunctivitis, often with pannus; tsa dra, interstitial keratitis and disciform stromal keratitis; and mu dip, a thin, blue film over the cornea that may not have a WM analog. Conjunctival squamous cell carcinoma, known as dey, and severe forms of spheroidal degeneration and gelatinous drop-like dystrophy, known as lingrow, are not treated with pha la, nor is nonseasonal conjunctivitis, known as mar task.

The more potent white form of pha la is not applied directly to the cornea, but reserved for severe shamdzir presentations, and often causes a significant burning pain when first applied. Dr. Yangkyi and her colleagues usually administer $4 \%$ topical lidocaine for anesthesia before applying the white pha la directly to the lesion. Often this pain becomes insignificant after a few applications of white pha $l a$, and prolonged treatment is continued without the need for anesthetic drops. Pterygium treatment may last several months, with daily application of the powder. The red form of pha la typically causes less pain and is used without anesthetic medication, even on the first use. Daily application for shorter lengths of time is the treatment for the other five disorders listed above. The therapy is complete when the patient and TTM doctor are satisfied with the outcome. Disabling side effects or complications are rarely seen, and unknown to the TTM doctors at 
Lhasa Menzikhang hospital. The basic ingredients of the red form of pha la are cotus root, camphor oil, cinnabar, dried fruit (Termalia chebula), and borate, all held together by crystal sugar. The precise preparation is described in detail in the TTM literature, and manufacture of both red and white pha la is complex, requiring a full day for a team of physicians. Approximately 100 new patients are treated and 3000 - 4000 pha la treatments are given annually at Lhasa Menzikhang Hospital. Only a few are patients with bacterial keratitis, and the second author did not observe treatment of these cases.

\section{Results}

Our first case to report is an 84 year old male monk concerned with ling tok, a corneal scar on his left eye. He had successful extra-capsular cataract surgery on the right eye at Menzikhang several years ago, and a history of a corneal abrasion on the left eye from a horsetail about fifty years ago. His visual acuity was 6/9 and 6/24 with correction. Slit-lamp examination showed pseudophakia OD, and a bandshaped $(2.1 \mathrm{~mm} \times 0.9 \mathrm{~mm})$ corneal scar and mild cataract in the left eye. No calcium was seen in this scar, but its location was suspicious for band-shaped keratopathy. Dilated fundus exam showed some mild age-related macular disease in both eyes. Red pha la was applied to the left cornea daily for 8 days, and microcystic edema was seen around the scar. At the end of treatment, best corrected vision OS was $6 / 9$. The scar size was measured as $1.4 \mathrm{~mm} \mathrm{x}$ $0.8 \mathrm{~mm}$, a small objective improvement that the authors believe exceeds the expected error of repeated measurement of a stable lesion.

Case two is a 58 year old male nomadic farmer, who had longstanding poor vision in his right eye and a new, visible lesion on the left eye. Visual acuity was no light perception in the right eye, $6 / 6$ without correction in the left. Slit-lamp examination showed a large spheroidal degeneration lesion (lingrow) centrally on the right cornea, as well as an opaque white cataract. There was no view of the retina and B-scan ultrasound was not available. The left eye showed a few small spheroidal lesions (ling tok) on the cornea, and a normal lens and retina. Red pha $l a$ was applied daily to the left cornea for 6 days. He was subjectively improved.
Case 3 is a 32 year old female, presenting with a multi-year history of shamdzir (pterygium) and associated symptoms. The lesion was $5 \mathrm{~mm}$ wide at the limbus and intruding $1 \mathrm{~mm}$ on the right cornea. From a WM perspective, the remainder of her eye examination, including refraction, slit-lamp and dilated fundus examination, were normal.

Dr. Yangkyi selected the red form of pha la to be applied to the lesion daily for several months. The applied medication is shown in Figure 1.

Case four is a 28 year old female with a multi-year history of corneal ling tok, a foreign body scar in her left eye. Vision was $6 / 6$ and $6 / 12$ and the scar dimensions were measured as $0.4 \mathrm{~mm}$ tall and 0.2 $\mathrm{mm}$ wide with a slight iron color present. The remainder of her WM eye examination was normal. Red pha la was applied daily to the lesion for 8 days with no objective change in size or appearance.

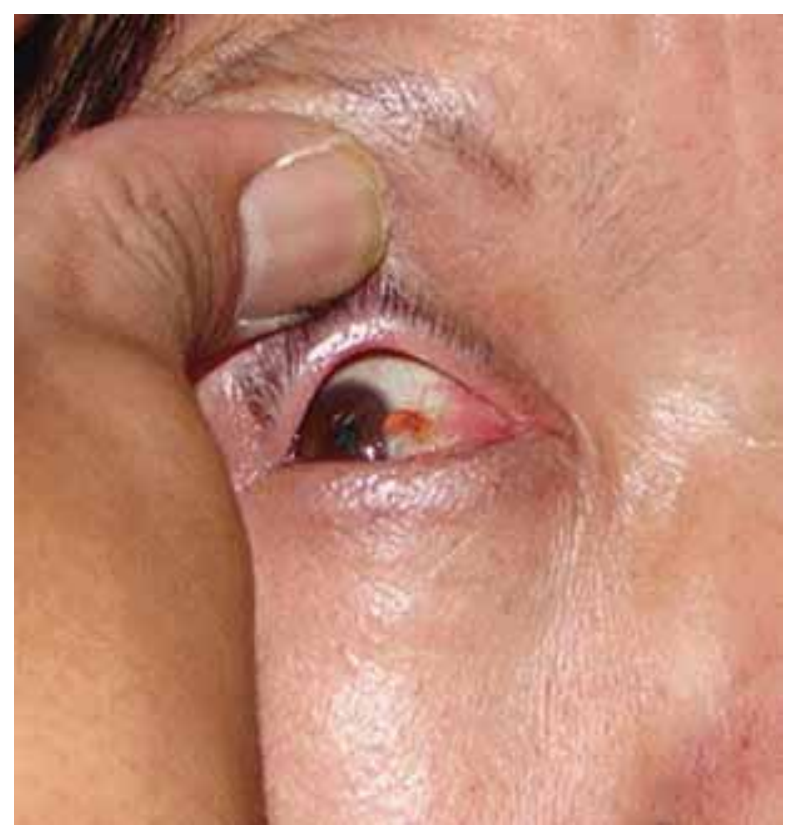

Figure 1: Application of pha la to the eye 
Conclusions: The outcome of the first case, from a WM perspective, shows objective improvement in the size and appearance of one of the lesions, contrary to what one would expect from the natural history of a long-standing corneal scar. The other cases were stable without complications. The outcomes, from a traditional Tibetan perspective, are positive because each patient believes that his disease and the future likelihood of requiring surgery has been reduced.
The authors hope to cooperate in the future for a larger randomized, controlled trial of the use of pha la, which has no WM analog. Many of these disorders, whose WM etiologies range from infectious and inflammatory to degenerative and dystrophic, are not considered amenable to medication in WM. If pha la treatment were to pass rigorous WM clinical scientific study for one or more of the diseases listed, it could add measurably to WM ophthalmologic capabilities. 